\title{
Detection of optic nerve involvement in retinoblastoma with enhanced computed tomography
}

\begin{abstract}
Purpose The study was designed to evaluate the value of enhanced computed tomography (CT) as a non-invasive test in the detection of optic nerve invasion in retinoblastoma. Methods Nineteen eyes in 17 consecutive retinoblastoma patients underwent $\mathrm{CT}$ studies performed with high spatial resolution $(512 \times 512)$ and $1.5 \mathrm{~mm}$ sections, both with enhancement and without enhancement. If the central retinal vessels (CRV) were subjectively visualised with intravenous enhancement, the optic nerve was considered to be free of retinoblastoma. Nineteen enucleated globes were processed for histopathology, and the optic disc and nerve examined with light microscopy for the presence or absence of tumour and the level of involvement. Results The correlation between the visualisation of CRV and the presence or absence of optic nerve involvement histopathologically was found to be highly significant ( $p=0.0006$, Fisher exact test). Conclusion In high spatial resolution CT with enhancement and $1.5 \mathrm{~mm}$ or thinner sections, non-visualisation of the CRV appears to be a reliable indicator of optic nerve invasion with retinoblastoma.
\end{abstract}

Key words Retinoblastoma, Computed tomography $(\mathrm{CT})$, Enhanced CT, Optic nerve, Central retinal vessels

The diagnosis of retinoblastoma in a great majority of cases is made primarily by indirect ophthalmoscopic examination, with ultrasonography used as a confirmatory procedure. Since most intraocular retinoblastomas contain calcium, echography is an ideal method for imaging these tumours. Even a small amount of calcium produces a significantly high internal reflectivity in echography. ${ }^{1,2}$ However, once retinoblastoma infiltrates the optic nerve or extends into the orbit through the sclera, echography becomes ineffective because of shadowing artifact from intraocular calcification and the non-calcified nature of extraocular tumour formation.

Consequently, other imaging procedures such as computed tomography (CT) and magnetic resonance imaging (MRI) are considered better modalities for evaluation of extraocular extensions of retinoblastoma. ' Of the two imaging procedures, $\mathrm{CT}$ is the preferred modality because of MRI's relative insensitivity to calcification. ${ }^{3.4}$ Both imaging procedures are valuable in detecting the presence of associated midline brain lesions ('trilateral' retinoblastoma). ${ }^{5,6}$

The involvement of the optic nerve indicates poor prognosis in retinoblastoma; therefore, special attention is directed to investigation of the optic disc area with imaging procedures.' In this study, we evaluated the role of enhanced $\mathrm{CT}$ to demonstrate the involvement of the optic nerve.

\section{Subjects and methods}

Seventeen patients with retinoblastoma were enrolled for the study in a consecutive fashion; 2 children with bilateral disease were included. Nineteen eyes were studied with CT of the globe and orbit with a GE-9800 CT unit. Scans were performed with high spatial resolution $(512 \times 512)$ and $1.5 \mathrm{~mm}$ sections overlaid from $0.5 \mathrm{~mm}$ to allow quality reformations. The cases were also studied with $1.5 \mathrm{~mm}$ sections after injection of intravenous contrast medium (Ultravist-300, $2 \mathrm{ml} / \mathrm{kg}$ ). Multiplanar reformations were obtained in enhanced scans. Special attention was paid to visualising central retinal vessels (CRV). If the central area of the optic nerve was enhanced anteriorly with intravenous contrast, CRV enhancement was labelled as 'present' (Fig. 1); if not visualised, it was labelled as 'absent' (Fig. 3A; Table 1). If the entire optic nerve was enhanced diffusely with contrast injection, not only CRV but the entire nerve were considered to be positive. These categories were based on subjective
C. Jacquemın

Z.A. Karcioglu

Kıng Khaled Eye Specialist Hospitat

c/o Medical Library

PO Box 7191

Riyadh 11462

Kingdom of Saud Arabia

Tel: 966-1-482-1234, x 1362

Fax: 966-1-482-1908 


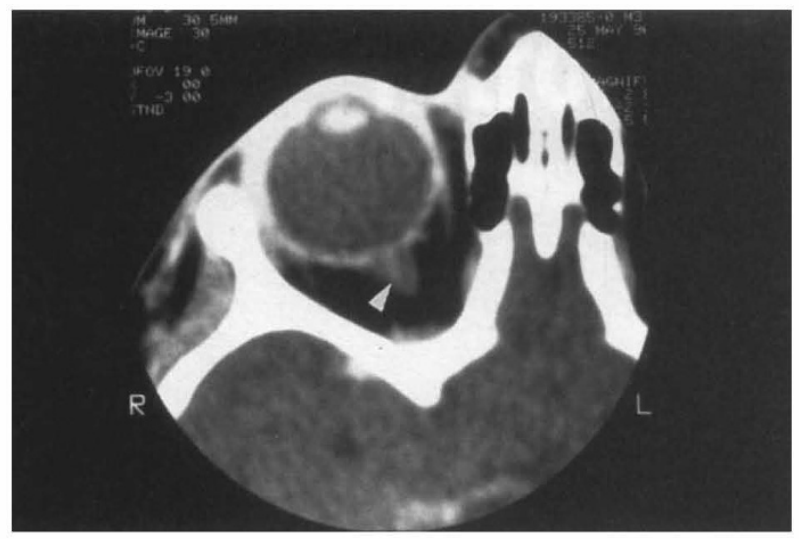

Fig. 1. Depiction of enhanced central retinal vessels in a normal globe without the presence of tumour (arrowhead).
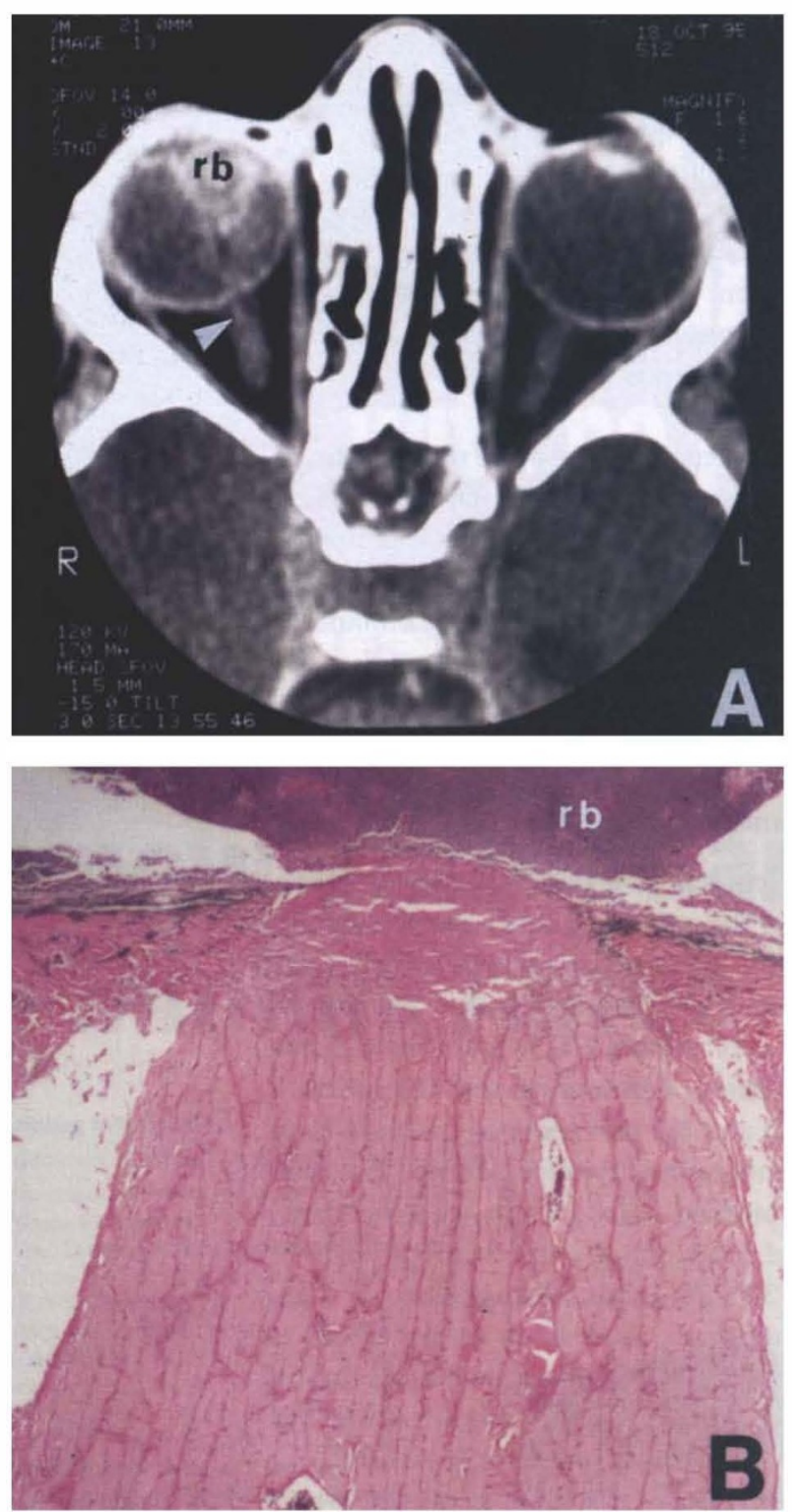

Fig. 2. (A) Depiction of the enhanced central retinal blood vessels (arrowhead) in a globe that contains retinoblastoma ( $r b$ ) without optic nerve head invasion. (B) Histopathological appearance of the same globe depicting non-involvement of the optic nerve head with retinoblastoma $(r b)$ (haematoxylin-eosin; original magnification $\times 2$ ). interpretation done in masked fashion by the radiologist (C.J.) and the ophthalmologist (Z.A.K.).

Nineteen globes were enucleated, fixed in $10 \%$ formaldehyde, and processed and stained in routine fashion for histopathological examination. Multiple $4-\mu \mathrm{m}$-thick sections through the optic disc, stained with haematoxylin-eosin, were studied by light microscopy in masked fashion. The optic disc was examined in at least six sequential sections for the presence or absence of retinoblastoma. If tumour was identified within the nerve fibre layer of the optic nerve head, the case was considered positive (anterior or posterior to lamina cribrosa) for optic nerve involvement; if not, it was considered negative (Table 1; Figs. 2, 3).

BMDP software was used for the statistical analysis.

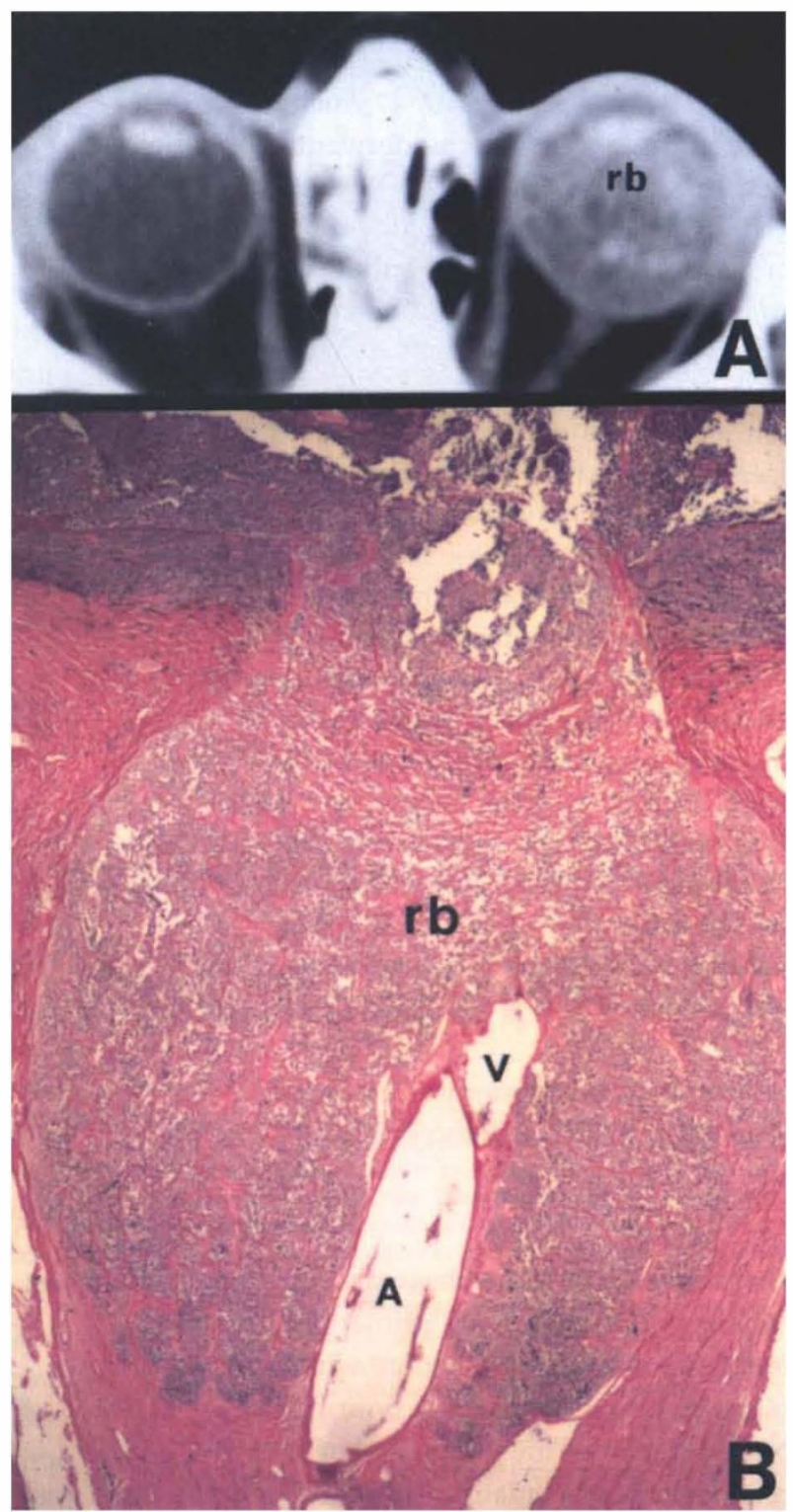

Fig. 3. (A) Non-visualisation of the central retinal blood vessels in a case with involvement of the optic nerve head with retinoblastoma (rb). (B) Histopathological depiction of the same globe showing the anterior segment of the optic nerve invaded by retinoblastoma $(r b)$. Note the irregular distentions of the central retinal artery $(A)$ and vein $(V)$ (haematoxylin-eosin; original magnification $\times 2$ ). 


\section{Results (Table 1)}

Of 19 eyes, CRV enhancement was present in $8(42.1 \%)$, absent in $8(42.1 \%)$, and questionably present in 3 $(15.8 \%)$. Optic nerve enhancement was present in 3 $(15.8 \%)$ and absent in $16(84.2 \%)$ eyes.

On histopathological examination of 19 enucleated globes, optic nerve involvement was negative in 10 $(52.6 \%)$. Retinoblastoma was present anterior to the lamina cribrosa in $1(5.3 \%)$ eye and posterior to the lamina cribrosa in $8(42.1 \%)$.

The correlation between the presence of CRV enhancement on CT and histopathological optic nerve involvement was studied in 19 eyes and found to be significant. All $8(100 \%)$ cases in which CRV enhancement was absent showed histopathological tumour involvement posterior to the lamina cribrosa. Of 8 cases in which CRV enhancement was present, 7 $(87.5 \%)$ eyes histopathologically revealed noninvolvement of the optic nerve and in $1(12.5 \%)$ case the optic nerve was involved with tumour anterior to the lamina cribrosa.

The correlation between visualisation of CRV on enhanced CT scans and histopathological optic nerve involvement (positive or negative) was highly significant $(p=0.0006$, Fisher exact test), whereas correlation between CRV visualisation on enhanced CT scans and choroidal involvement on histopathology was found to be statistically insignificant $(p=0.14)$.

\section{Discussion}

During investigations for retinoblastoma, the two main aims are to establish the diagnosis and to determine the extent of the tumour. ${ }^{79}$

Most retinoblastoma patients present with leucocoria and ophthalmoscopic recognition of retinoblastoma. In a small percentage of cases, however, other conditions that cause leucocoria, such as congenital cataracts, toxocariasis, retinopathy or prematurity, persistent hyperplastic primary vitreous and Coats disease, may be confused with retinoblastoma." A number of modalities, including ultrasonography, CT and MRI, are helpful in establishing the diagnosis. Although CT is capable of detecting calcifications within retinoblastoma, ultrasonography is more discriminating, particularly in visualising calcium within small tumours. ${ }^{6.11}$ Because of the frequency of tumour calcification, ultrasonography is considered the most sensitive and practical test for confirming the diagnosis. ultrasonography are that it is non-invasive, there is no radiation exposure, and, unlike other imaging procedures, it can be quickly and inexpensively done in the office.

Once the diagnosis is established, the next step is to determine the boundaries of the tumour within the eye and whether there are extensions into the optic nerve, sclera, and beyond the globe. For the latter purpose, CT and MRI are superior to ultrasonography because they offer better marginal detail and are not affected by artifactual shadowing due to intraocular calcification.

MRI offers better contrast resolution and therefore more information regarding the size, shape and margins of the tumour. ${ }^{313}$ The superior contrast resolution of MRI provides a better ability to differentiate tumour tissue from subretinal fluid, resulting in greater accuracy in delineating the tumour. ${ }^{1+15} \mathrm{~A}$ recent review by Davis and Newman ${ }^{10}$ advised the use of ultrasonography as the initial imaging modality to assess the globe and the 'immediate retro-ocular orbit', with CT as the secondary imaging modality. Gadopentetate dimeglumine (Gd-DPTA)-enhanced MRI is also reported to be useful in evaluating optic neuropathies/injuries and assessing the optic nerve in retinoblastoma cases. ${ }^{17-19}$ Intravenous enhanced MRI provides good delineation of the tumour from adjacent fluid medium, better detailing of tumour

Table 1. Summary of CT and histopathology data in 19 e'yes

\begin{tabular}{|c|c|c|c|c|}
\hline Case no. & $\begin{array}{l}\text { CRV enhancement } \\
\text { (present / absent / questionable) }\end{array}$ & $\begin{array}{l}\text { ON enhancement } \\
\text { (present / absent) }\end{array}$ & $\begin{array}{l}\text { ON involvement } \\
\text { (negative/ant. LC/post. LC) }\end{array}$ & Tumour histology \\
\hline 1 & Absent & Absent & Post. LC & Poorly differentiated, $++\mathrm{Ca}$ \\
\hline 2 & Present & Absent & Negative & Poorly differentiated \\
\hline 3 & Absent & Present & Post. LC & Diffuse ne'crosis \\
\hline 4 & Present & Absent & Negative & Poorly differentiated \\
\hline 5 & Absent & Absent & Post. LC & Extrascleral nodule \\
\hline 6 & Questionable & Absent & Negative & Diffuse necrosis \\
\hline 7 & Absent & Present & Post. LC & Poorly differentiated, necrotic, $+\mathrm{Ca}$ \\
\hline 8 & Absent & Absent & Post. LC & Poorly differentiated, $++\mathrm{Ca}$ \\
\hline 9 & Absent & Absent & Post. LC & Diffuse necrosis, $++\mathrm{Ca}$ \\
\hline 10 & Present & Absent & Negative & Diffuse necrosis, $+++\mathrm{Ca}$ \\
\hline $11 \mathrm{OS}$ & Present & Absent & Negative & Well differentiated, $++\mathrm{CA}$ \\
\hline OD & Present & Absent & Ant. LC & Well differentiated \\
\hline 12 & Present & Absent & Negative & Necrosis, $++\mathrm{Ca}$ \\
\hline 13 & Present & Absent & Negative & Necrosis, $++\mathrm{Ca}$ \\
\hline 14 & Present & Absent & Negative & Poorly differentiated \\
\hline $15 \mathrm{OS}$ & Questionable & Absent & Negative & Well differentiated \\
\hline OD & Questionable & Absent & Negative & Well differentiated \\
\hline 16 & Absent & Present & Post. LC & Poorly differentiated \\
\hline 17 & Absent & Absent & Post. LC & Well differentiated \\
\hline
\end{tabular}

CRV, central retinal vessels; $\mathrm{ON}$, optic nerve; $\mathrm{Ca}$, calcium $(+,++$ or +++$)$. 
vascularity, and better definition of orbital blood vessels such as the superior ophthalmic vein. But there are also limiting factors for studying the optic nerve head with MRI, including poor signal-to-noise ratio, reduced spatial resolution, and thicker sections (usually $3 \mathrm{~mm}$ ) and interslice intervals with spin echo sequences. The most serious shortcoming of MRI in retinoblastoma cases is its relative insensitivity towards calcification. ${ }^{420}$ On the other hand, calcification can be detected by CT with a high degree of accuracy in approximately $90 \%$ of cases. ${ }^{15}$ Further advantages of CT are its easy enhancement capability and its potential for detecting the presence of calcified midline brain lesions.

Significant improvements in CT technology have increased its spatial and density resolution as well as its ability to obtain direct volumetric acquisitions (spiral CT). Cuts are possible in a short acquisition time, as well as quality angiograms. ${ }^{21,22}$ These technical improvements have significantly aided retinoblastoma diagnosis and management. Spiral CT studies are favoured over MRI by some due to relatively easier access and lower cost, with MRI studies being reserved for more difficult cases and when better intracranial or meningeal studies are needed. ${ }^{21,22}$ The short acquisition time of orbital CT studies (seconds) compared with MRI (minutes) decreases motion artifact.

Although the literature is in consensus that highresolution $\mathrm{CT}$ is a very useful adjunct for delineating the size and location of retinoblastoma, few investigators have studied the detection of optic nerve involvement using CT, with or without enhancement. One study by John-Mikolajewski et al. ${ }^{23}$ examined this topic and declared that retinoblastoma extension through the lamina cribrosa of the optic nerve could not be determined by CT; however, only 3 of 63 CT scans with enhancement were studied in their series. The unconvincing conclusion of the above-cited report, coupled with a sparsity of studies in the literature, prompted us to undertake this study to correlate the findings of enhanced CT images with post-enucleation histopathology.

Using ultrathin $(1.5 \mathrm{~mm})$ sections to evaluate structures with a density significantly different from adjacent tissues, our results indicated improved visualisation of the CRV. Although thin sections lead to low contrast resolution, this was not a disadvantage in our study because contrast enhancement was used. The combination of ultrathin sections and contrast injection enabled us to detect significant density differences between CRV and optic nerve tissue.

In 8 cases where tumour was posterior to the lamina cribrosa, architectural disruption of the central retinal vessels with tortuosity and distention could be visualised histopathologically. This was not surprising considering the anatomy of the optic disc and anterior optic nerve area and the rigid surroundings of the central retinal vessels as they pass through the lamina cribrosa. Most authorities accept the presence of an elaborate vascular network between central retinal and posterior ciliary vessels and the pial vascular system in the anterior optic nerve region. ${ }^{24,25}$ Any mass formation in the area, such as a bulk of retinoblastoma and/or surrounding oedema, can easily produce distortion of the anastomotic network with or without direct compression on the central retinal artery and vein, leading to non-visualisation of these vessels. The tortuosity and distention of CRV that we observed on histological sections were obviously secondary to mechanical obstruction created by the mass effect. Even if CRV are not completely occluded by the pressure of the tumour, disruption of blood flow within the anterior optic nerve microvasculature may be enough to distort the CT appearance. ${ }^{26}$

Our study concluded that in high spatial resolution, enhanced CT with $1.5 \mathrm{~mm}$ sections, non-visualisation of the CRV reliably indicates optic nerve invasion with retinoblastoma. Although advances in CT and MRI angiography, echo planar techniques and magnetic resonance spectroscopy - may eventually offer better and safer imaging modalities, it seems that utilisation of enhanced CT with ultrathin sections is a reliable and practical addition to our current armamentarium for retinoblastoma management.

\section{References}

1. Byrne SF, Green RL. Ultrasound of the eye and orbit. St Louis: Mosby, 1992:196-200.

2. Shields JA, Michaelson JB, Leonard BC, et al. B-scan ultrasonography in the diagnosis of atypical retinoblastomas. Can J Ophthalmol 1976;11:42-51.

3. Mafee MF, Goldberg MG, Greenwold JM, et al. Retinoblastoma and simulating lesions: role of CT and MR imaging. Radiol Clin North Am 1987;25:667-82.

4. Wilms G, Marchal G, Von Fraeyenhoven L, et al. Shortcomings and pitfalls of ocular MRI. Neuroradiology 1991;33:320-5.

5. Blach LE, McCormick E, Abramson EH, Ellsworth RM. Trilateral retinoblastoma - incidence and outcome: a decade of experience. Int J Radiat Oncol Biol Phys 1994;29:729-33.

6. Nucci P, Modorati G, Pierrol L, et al. Comparative evaluation of echography and CAT in diagnosis of retinoblastoma. Minerva Pediatr 1989;41:129-31.

7. Kopelman JE, McLean IW, Rosenberg SH. Multivariate analysis of risk factors for metastasis in retinoblastoma treated by enucleation. Ophthalmology 1987;94:371-7.

8. Shields JA, Shields CL. Intraocular tumours: a text and atlas Philadelphia: Saunders, 1992:341-62.

9. Karcioglu ZA, Al-Mesfer SA, Jabak MH, et al. Workup for metastatic retinoblastoma: a review of 261 patients. Ophthalmology 1997;104:307-12.

10. Gupta KL, Righi AM, Mihara F, et al. MRI, ultrasound and surgical correlation of orbital tumours. Neuroradiology 1991;33(Suppl):71.

11. Shields JA, Stephens RF. Ultrasonography in pediatric ophthalmology. In: Harley RD, editor). Pediatric ophthalmology. Philadelphia: Saunders, 1982:145-54.

12. Shields JA, Michaelson JB, Leonard BC, et al. Retinoblastoma in an 18-year-old male. J Pediatr Ophthalmol 1976;13:275-7.

13. Haik BG, St Louis L, Smith ME, et al. Magnetic resonance imaging in the evolution of leucocoria. Ophthalmology 1985;92:1143-52.

14. Wilms G, Dralands G, Fraeyenhoven L, et al. Magnetic resonance imaging in lesions of the eye globe. J Belge Radiol $1989 ; 72: 1965-72$. 
15. Beets-Tan RGH, Hendriks MJ, Ramos LMP, Tan KEW. Retinoblastoma: CT and MRI. Neuroradiology 1994;36:59-62.

16. Davis PC, Newman MJ. Advances in neuro-imaging of the visual pathways. Am J Ophthalmol 1996;121:690-705.

17. Guy J, Mancusco A, Quisling RG, et al. Gadolinium-DPTAenhanced magnetic resonance imaging in optic neuropathies. Ophthalmology 1990;97:592-600.

18. Takehara S, Tanaka $T$, Uemura $K$, et al. Optic nerve injury demonstrated by MRI with STIR sequences. Neuroradiology 1994;36:512-4.

19. Ainbinder DT, Haik BG, Frei DF, et al. Gadolinium enhancement: improved MRI detection of retinoblastoma extension into the optic nerve. Diagn Neuroradiol 1996;38:778-81.

20. Weber AL, Mafee MF. Evaluation of the globe using computed tomography and magnetic resonance imaging. Isr J Med Sci 1992;23:145-52.
21. Hermans R, Marchal G, Feenstra L, Baert AL. Spiral CT of the temporal bone: value of image reconstruction of submillimetric table increments. Neuroradiology 1995:37:150-4.

22. Katz DA, Marks MP, Napel SA, et al. Circle of Willis: evaluation with spiral CT angiography, MR angiography, and conventional angiography. Radiology 1995;195:445-9.

23. John-Mikolajewski V, Messmer E, Sauerwein W, Feundlieb O. Orbital computed tomography. Does it help in diagnosing the infiltration of choroid, sclera, and/or optic nerve in retinoblastoma? Ophthalmic Pediatr Genet 1987;8:101-4.

24. Hayreh SS. Structure and blood supply of the optic nerve. In: Heilmann K, Richardson KT, editors. Glaucoma: conceptions of a disease. Philadelphia: Saunders, 1978:78-103.

25. Orgul S, Cioffi GA. Embryology, anatomy and histology of the optic nerve vasculature. J Glaucoma 1996;5:285-94.

26. Hayreh SS, Edwards J. Ophthalmic artery and venous pressures: effect of acute intracranial hypertension. $\mathrm{Br} \mathrm{J}$ Ophthalmol 1971;55:649-66. 